\title{
Circle Extraction via Least Squares and the Kalman Filter
}

\author{
Mark S Nixon \\ Dept. of Electronics and Computer Science, University of Southampton, Southampton, UK
}

\begin{abstract}
Two new techniques have been developed to extract circles in computer images and this paper clarifies their implementation. One technique uses nonlinear least squares, the other an extended Kalman filter. Parameter estimation is based on analysing the residual gradient direction where the locus of an approximation to a circle intersects the target circle. This approach allows powerful estimation techniques to be used for feature extraction in computer vision. The least squares technique is based on adapting an earlier method developed for ellipse extraction which has been modified not only for circle estimation but also to reduce sensitivity in parameter estimation. The Kalman filter algorithm is an extended version arranged to estimate the circle's parameters.

Simulation results show that both techniques can extract circles in application but the Kalman filter implementation took more iterations and a number of factors limit its use The least squares technique only is shown applied to grey level images. Both techniques serve to extract circles when an initial approximation to the circle is known and hence are most suited to tracking circles in computer images. The techniques are at present being extended to include data weighting according to edge magnitude and direction.
\end{abstract}

\section{Introduction}

Some techniques have already been developed to extract circles using least squares. One approach concerned iterative fitting of coordinate values to minimise the error between a set of given points and an estimated arc [1]. A non-iterative solution was formulated later by deriving a parameter set which minimises the difference between computed and predicted area using a modified mean square error function [2]. A recent survey of quadratic curve extraction [3] studied the accuracy of the parameters which describe quadratic curves and introduced a new error function to derive a measure of quality of the extracted parameters.

Both new techniques have been developed for circle extraction and use gradient direction information in a new way. A least squares technique for ellipse extraction [4] has been extended to circle extraction. Gradient direction information has also been used before to reduce data in circle extraction $[5,6]$ but these use measured edge direction alone. For circles, the edge direction can be arranged to point towards the centre of the circle and hence speed extraction of the centre coordinates. The new techniques are based not on using direction information alone, but on using the difference between the edge direction predicted from an approximation to a circle from the measured edge 
direction. At points where the target circle intersects with the approximation, the gradient direction can be predicted for the perimeter of the approximation using the equation which describes it. The edge gradient direction on the perimeter of the circle in the image can be measured using an edge detection operator. The difference between these gradient directions is intimately related to the error in the values used for the parameters of the approximation.

By combining the residual information at intersecting points, an iterative least squares algorithm has been developed to estimate parameter values. The Kalman filter algorithm also uses the discrepancy in edge direction information to correct parameter estimates and can produce estimates in a point by point iterative manner according to its basic formulation. It was included in this study to determine whether its formulation provided any benefits to circle extraction. These concern estimation in a direct iterative manner where the error covariance describes error associated with the estimation procedure. The error covariance is used to evaluate the Kalman gain which controls contribution of the residual gradient direction to the parameter estimates. A bias corrected extended Kalman filter for ellipse extraction [7] based on a linearisation of the maximum likelihood principle rather than coordinate data. This aimed to reduce bias to high curvature fits. Following iterative estimation of ellipse parameters the final error covariance was used to evaluate a confidence envelope for the fitted ellipse. Another approach [8], also based on coordinate data, extended arcs using a Mahalanobis distance measure to select segments of edge data to update the estimates from the Kalman filter. Using residual gradient direction required an extended Kalman filter because the measurement is not linearly related to the parameters which describe a circle. The formulation is standard but arranged for this application. Both techniques are demonstrated to work in simulation to extract a light disk from a dark background. The least squares technique is also shown applied to real images and research progresses to capitalise on using weighting according to edge direction consistency and on appropriate point selection for the Kalman filter algorithm.

\section{Least squares technique for circle extraction}

Given a circle defined by

$$
\left(x-x_{0}\right)^{2}+\left(y-y_{0}\right)^{2}=r^{2}
$$

where $x_{0}, y_{0}$ are the coordinates of the centre and $r$ is the radius. The gradient direction is given by differentiation, expressed in terms of the $x$ coordinate only as

$$
f(x, \underline{w})=-\frac{\left(x-x_{0}\right)}{\sqrt{r^{2}-\left(x-x_{0}\right)^{2}}}
$$

where $\underline{w}$ is a vector of the unknown parameters

$$
\underline{w}=\left\{x_{0} r\right\}
$$

The values of these unknown parameters is to be estimated and the true values (the target) are $\underline{w}$ and their estimates are $\underline{\hat{w}}$. The values of the starting set of estimates $\underline{\hat{w}}_{s}$ is corrected by increments $\underline{\delta} w$ to achieve a new set (or end set) of estimates $\underline{\hat{w}}_{e}$ 


$$
\text { i.e. } \underline{\hat{w}}_{e}=\underline{\hat{w}}_{s}+\underline{\delta} w
$$

where $\underline{\delta} w$ is the vector of increments to the parameter estimates, formed from $\delta x_{0}$ the increment to the $x$ coordinate of the centre and $\delta r$ the increment to the radius. The increments are calculated by combining residual gradient direction at intersecting points on the locus of the approximation and of the target circle by the method of least squares. The difference between the measured and predicted gradient direction is

$$
\tilde{f}(x, \underline{w})=f(x, \underline{w})-f(x, \underline{\hat{w}})
$$

where the predicted gradient direction is $f(x, \underline{\hat{w}})$ and $f(x, \underline{w})$ is the measured gradient direction at the point of interest. By expanding the predicted gradient direction to first order by Taylor series and by substitution in eqn. 4 the sum of differences squared, $D^{2}$, over $N$ matching points is

$$
D^{2}=\sum^{N}\left\{f(x, \underline{w})-f\left(x, \underline{\hat{w}}_{s}\right)-\frac{\partial f\left(x, \underline{\hat{w}}_{s}\right)}{\partial \underline{w}} \underline{\delta} w\right\}^{2}
$$

We seek to minimise this sum by best choice of values for the elements of $\underline{\delta} w$. By differentiation the result the solution is

$$
\underline{\delta} w=\alpha^{-1} \beta
$$

where the elements of $\alpha$ and $\beta$ are computed according to

$$
\begin{aligned}
\alpha_{j k} & =\sum^{N}\left\{\frac{\partial f\left(x, \underline{\hat{w}}_{s}\right)}{\partial w_{j}} \frac{\partial f\left(x, \hat{\underline{w}}_{s}\right)}{\partial w_{k}}\right\} \\
\beta_{k} & =\sum^{N}\left\{\left\{f(x, \underline{w})-f\left(x, \underline{\hat{w}}_{s}\right)\right\} \frac{\partial f\left(x, \underline{\hat{w}}_{s}\right)}{\partial w_{k}}\right\}
\end{aligned}
$$

Calculation starts by choosing values for the first approximation, $\underline{\hat{w}}_{s}$. At intersecting points between the two loci the edge gradient direction can be measured to provide values for $f(x, \underline{w})$, and can be predicted from Equation 2 to give values for $f\left(x, \underline{\hat{w}}_{s}\right)$. The predicted gradient direction is expressed as an angle, rather than as a fraction, to preserve isotropy and hence a balanced contribution of the residual gradient direction. The predicted gradient direction is then

$$
f(x, \underline{\hat{w}})=-\tan ^{-1}\left(\frac{\left(x-x_{0}\right)}{\sqrt{r^{2}-\left(x-x_{0}\right)^{2}}}\right)
$$

This provides edge direction as the angle between the $x$ axis and the tangent to the locus. The edge detection operator is then arranged to provide the edge direction in angular form, tangential to the edge. The difference in these angles is then used, together with the partial derivatives, to form the elements of the matrix $\beta$, Equation 7 . The partial derivatives themselves are used to calculate the matrix $\alpha$, Equation 6 . After the set of matching points has been exhausted, the increments to the first approximation can be 
evaluated, Equation 5. The new approximation $\underline{\hat{w}}_{e}$ can then be calculated according to Equation 3. This then forms a new approximation for subsequent estimation, and estimation proceeds in an iterative manner.

The procedure has not involved calculation of a value for the $y$ coordinate of the centre, $y_{0}$. In the previous development the gradient direction was reformulated to exclude the coordinate $x_{0}$, which for a circle would be in terms of $y_{0}$ and $r$ only and with a parallel set of equations to estimate values for $\underline{w}^{\prime}=\left\{y_{0} r\right\}$. However, choice of minimising an error measure expressed in terms of $d y / d x$ only does not penalise situations where the $y_{0}$ coordinate is at some distance from its target value and where the other two parameters are correct, since in this situation the difference in gradient direction difference would contribute less to correct $y_{0}$. The inverse, $d x / d y$, does however provide much greater information in this case, since the gradient difference depends directly on the error in the estimate of $y_{0}$. Accordingly the algorithm has been modified to search for $\underline{w}^{\prime}$ where the gradient direction difference and gradient direction are then

$$
\begin{gathered}
\tilde{f}_{2}\left(y, \underline{w}^{\prime}\right)=f_{2}\left(y, \underline{w}^{\prime}\right)-f_{2}\left(y, \underline{\hat{w}}^{\prime}\right) \\
f_{2}\left(y, \underline{w}^{\prime}\right)=\frac{-\left(y-y_{0}\right)}{\sqrt{r^{2}-\left(y-x_{0}\right)^{2}}}
\end{gathered}
$$

with suitable reformulation of Eqns 4-7. This gradient direction is again measured as an angle as in Eqn. 8 and therefore also required modification of the edge detection operator to provide edge direction consistent with their prediction. Expressing the edge direction using angular measurements preserves isotropy in the residual. This is equal in both measurements since one angle is a reflection of the other. This modification has improved consistency in extraction of the $y$ centre coordinate alone. The radius estimate is the mean of its two estimates.

\section{Kalman filter for circle extraction}

The Kalman filter is now well established in many applications. These include adaptive versions which are tailored not only to reduce the effect of noise on signals but also to determine the parameters which describe them. In this application we seek to estimate the parameters of a circle. The notation used is the same as for least squares, save that estimation is iterative. We seek to estimate $\underline{\hat{w}}_{n+1}$ based on the current estimates $\underline{\hat{w}}_{n}$ and the edge direction at point $n$. A basic Kalman filter algorithm cannot be used since the gradient direction nonlinear and is replaced in the Kalman filter algorithm by a Jacobean vector $H_{n}$

$$
H_{n}=\left[\begin{array}{ll}
\frac{\partial f\left(x_{n}, \underline{\hat{\hat{w}}}\right)}{\partial x_{0}} & \frac{\partial f\left(x_{n}, \underline{\hat{w}}\right)}{\partial r}
\end{array}\right]^{T}
$$

The Kalman filter algorithm is then 


$$
\begin{aligned}
K_{n} & =P_{n} H_{n}^{T}\left(H_{n} P_{n} H_{n}^{T}+R\right)^{-1} \\
\underline{\hat{w}}_{n+1} & =\underline{\hat{w}}_{n}+K_{n}\left(f\left(x_{n}, \underline{w}\right)-f\left(x_{n}, \underline{\hat{w}}\right)\right) \\
P_{n+1} & =P_{n}-K_{n} H_{n} P_{n}
\end{aligned}
$$

where $f(x, \underline{w})$ denotes the true gradient information as earlier, $K_{n}$ is the Kalman gain and $P_{n}$ is the error covariance. The equation to update the error covariance is omitted since parameters only are estimated and the state transition matrix is then unity and there is no process noise contaminating the parameters. The algorithm requires initial values for $P_{0}$ and the measurement noise covariance, $R$. These may be considered as tuning factors but effectively describe the expected error in the initial approximation, the initial values chosen for the parameters, $\underline{\hat{w}}_{0}$ and were chosen as

$$
\begin{aligned}
P_{0} & =\left[\begin{array}{ll}
5.0 & 1.0 \\
1.0 & 5.0
\end{array}\right] \\
R & =[0.5]
\end{aligned}
$$

The elements of $P_{0}$ reflect an equal uncertainty in the values for the centre coordinate and the radius. Statistical cross-correlation between the uncertainty in each of these parameters is reflected in the off-diagonal elements. The measurement noise covariance appears suitable for the errors associated with the edge detection algorithm. Estimation proceeds in an iterative manner following choice of the initial approximation. At each point the difference between the expected and measured gradient direction is used to correct the parameter estimates. As in the least squares algorithm, a parallel algorithm is used to estimate the $y$ centre coordinate and radius, which is again based on $d x / d y$. The edge direction processes are then the same as for the least squares algorithm. The Kalman filter algorithm operates in a point-iterative manner around the locus of intersection. When all intersecting points have been analyzed, the new parameters are evaluated according to eqn. 3 , and estimation continues along the intersection trajectory of the circle described by the new parameters.

\section{Results}

\subsection{Simulation results}

Each technique was assessed in simulation by using a white circular disc on a dark background as the target. In the simulation studies the edge direction data was provided by a least squares algorithm. Following specification of the initial approximation, estimation proceeded in an iterative manner where the parameter estimates after one iteration were used to prime the procedure for the following iteration. Both techniques finished either when the increments were sufficiently small (and parameters did not change between successive iterations) or when there were insufficient intersecting points. Example estimation runs are shown Fig. 1 where (a) is the least squares technique and (b) the Kalman filter, where both start from the same initial approximation. The perimeter of the target 
circle was described by centre coordinates $x_{0}=45$ and $y_{0}=62$ and the radius $r=39$. Fig. 1 shows the target disc together with (black) circles representing the result of each iteration of each technique. The parameters describing the initial approximation were $\hat{x}_{0}=42, \hat{y}_{0}=73$ and $\hat{r}=29$. This can be seen to intersect the target towards the right hand side. By least squares, the first iteration resulted in a new set of estimates $\hat{x}_{0}=45, \hat{y}_{0}=65$ and $\hat{r}=43$. This resulted in a circle which is clearly much closer to the target. Estimation then proceeded in this iterative manner and the fourth iteration resulted in increments which were sufficiently small to warrant termination and the final values were $\hat{x}_{0}=45, \hat{y}_{0}=63$ and $\hat{r}=40$. Note that after the second iteration the estimated circle was larger than the target and the estimates were corrected to converge to the target circle.

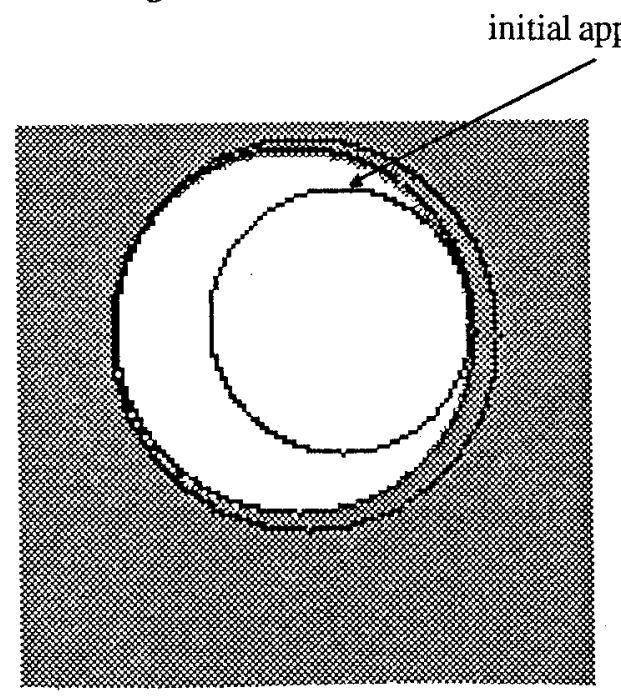

(a)

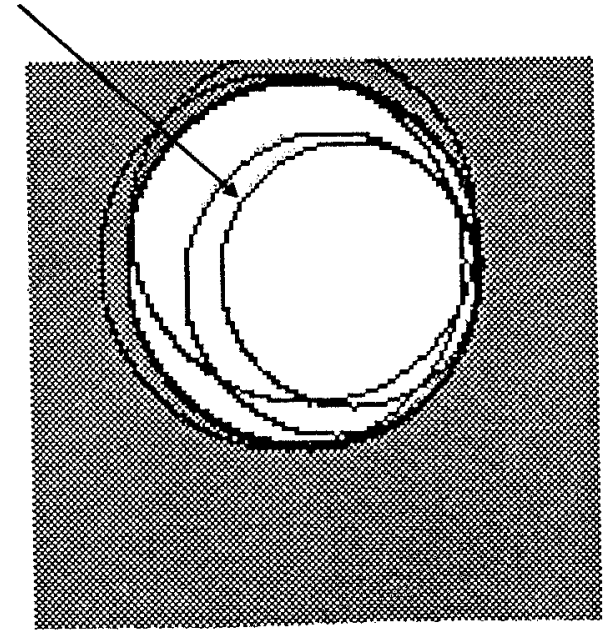

(b)

Figure 1 Simulation Results (a) Least Squares (b) Kalman filter

The sum of differences squared between the true value of each parameter and its estimate gives an error measure $c$

$$
c=\left(x_{0}-\hat{x}_{0}\right)^{2}+\left(y_{0}-\hat{y}_{0}\right)^{2}+(r-\hat{r})^{2}
$$

Fig. 2 shows the error measure reducing for a series of estimation runs, (a) is for least squares and (b) for the Kalman filter. These are actually two groups of four estimation runs where each group started for an approximation of the same radius, with initial centre coordinates given by reflection about the target centre in each of the four quadrants. For least squares, all eight runs can be seen to converge to close to the target with small error. The error measure does increase on three occasions but then decreases illustrating recovery. The divergence appears due to quality of the edge direction data. The Kalman filter algorithm has also been applied in simulation to extract the same circle from the same initial approximations used for the least squares technique. Again, successive improvement in the approximation delivered by the Kalman is illustrated in Fig. 1(b) 
which shows the Kalman filter algorithm successfully estimating both coordinates of the centre, together with the radius, aiming for the same target as in Fig. 1(a) and using the same initial approximation. After the fifth iteration the error measure was reduced to 1 pixel $^{2}$ where the $y$ centre coordinate was 1 pixel in error and the other two parameters were correct.

There are a number of aspects specific to the Kalman filter implementation. The specification of initial error covariance is inherent to the Kalman filter algorithm, eqn. 15. These elements are chosen as a pessimistic expectation of the error in the initial approximation. The error covariance contributes to the Kalman gain and controls convergence of the parameter estimates. Too large values can cause over-reliance on measurement values whereas when the Kalman gain is small, the measurement contributes little to update the parameter estimates. To ensure that optimal estimation is achieved, it would then appear necessary to choose appropriate values for these parameters for each estimation run but this is impractical and so for the series of simulation tests fixed values were used.

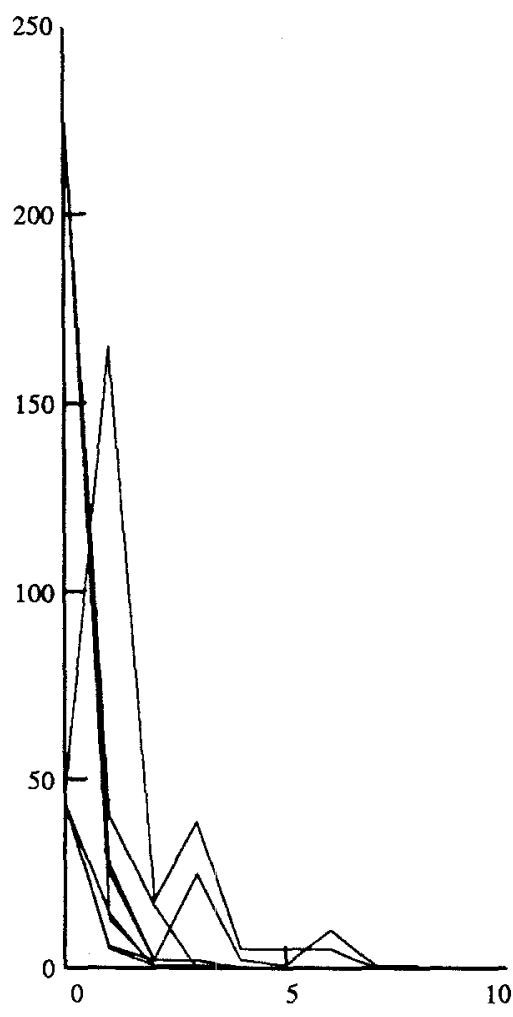

(a)

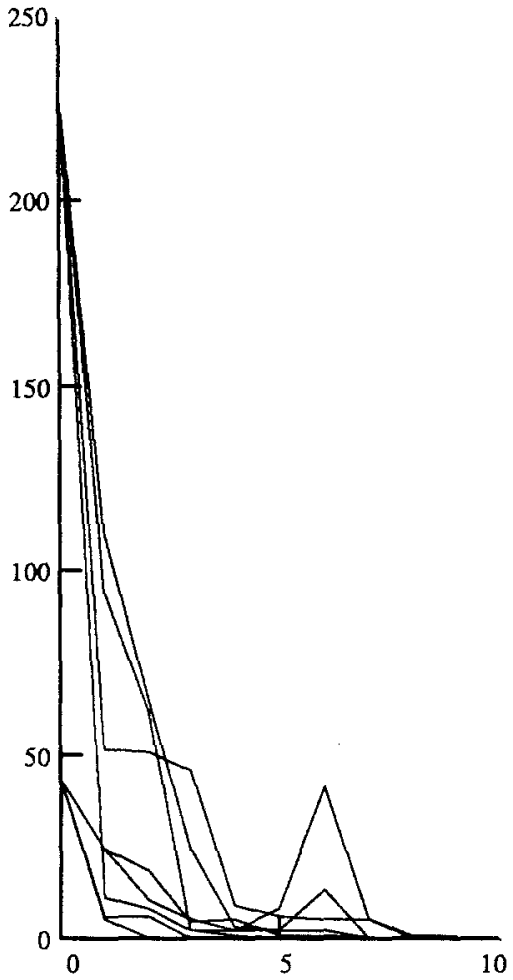

(b)

Figure 2 Error Measure Reduction in Simulation (a) Least Squares (b) Kalman Filter 
The effect on the performance error for a selection of estimation runs starting from the same eight points is shown in Fig. 2(b) where the vertical axis is the error measure, and the horizontal axis is the iteration no. However these results for the Kalman filter required 41 iterations in total for the eight initial approximations whereas application of the least squares technique with the same eight initial approximations required 27 iterations. The average improvement (reduction) in the error measure for each iteration was 27 pixels $^{2}$ for the Kalman filter algorithm compared with 41 pixels $^{2}$ for the least squares technique (with standard deviation 51 and 83 pixels respectively). Comparison of Fig. 1(a) with 1(b) shows the least squares technique requiring only two iterations to converge to the target circle whereas the Kalman filter algorithm required five. This reflects the general observed performance of the two algorithms, namely that the Kalman filter algorithm required more iterations to converge to the target.

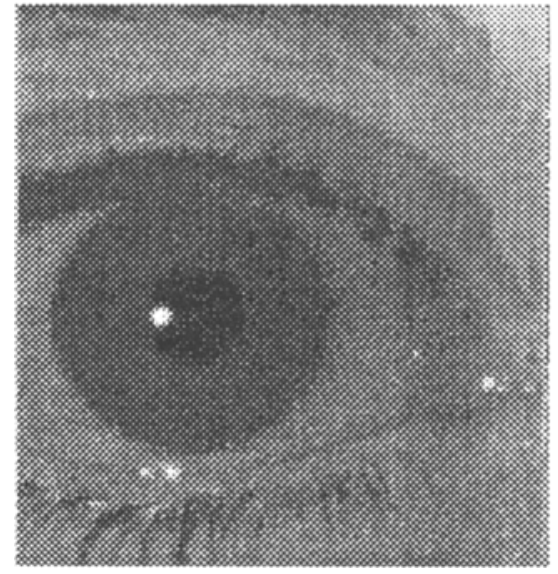

(a)

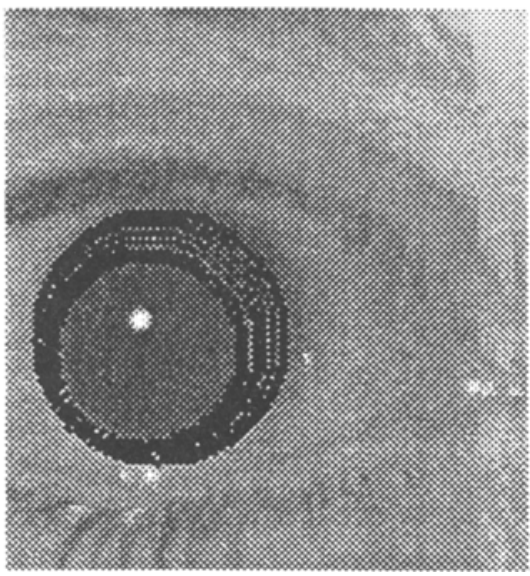

(b)

Figure 3 Eye Image (a) and Iris Extraction (b)

It is possible to control the convergence of the Kalman filter algorithm by different selection of the values for error covariance and noise covariance to prime estimation, but it proved impossible to choose values which consistently used less iterations than the least squares algorithm. Also, a main virtue of the least squares algorithm in comparison with the Kalman filter is that it needs none of these parameters to be specified. For these reasons the least squares algorithm appears the most practicable of the two techniques and hence only was tested on real images.

\subsection{Application to grey level images}

Illustrative applications with real images have demonstrated that the least squares technique can operate in a real environment where there is occlusion and data segments are missing. In application to real images, the edge direction data was provided by a version of the Canny operator applied to process $128 * 128$ 8-bit images. The edge data was then thresholded according to edge magnitude. Figure 3 shows a human eye with a sequence of iterations to estimate the position of the iris. In Fig. 3(b) a smaller circle 
is used as the first approximation which then homes in on perimeter of the iris. Note that the convergence trajectory passes through (and ignores) the pupil which presents another, small, circle. The technique can bypass small circles due to the preponderance of edge points associated with the larger target circle in the region of intersection. The circle extracted by the technique closely matched the largest contiguous circle derived by applying a Hough transform.

\section{Conclusions}

Basing feature extraction on residual gradient direction allows estimation techniques to be developed to locate circles in images. A least squares technique has been based on an earlier method for ellipse extraction with particular modification to improve sensitivity in the estimation of one parameter. A Kalman filter formulation required extension to handle the measurement nonlinearity and can be used to estimate circle parameters in a point-iterative manner.

Simulation studies emphasised difficulty in appropriate implementation of the Kalman filter and in selection of parameters used to prime its operation. Though the Kalman filter algorithm can be arranged to determine circle parameters it usually required more iterations and so the least squares technique only was applied to real images and is demonstrated in use to locate the perimeter of the iris of a human eye.

\section{References}

1. U. M. Landau: Estimation of a circular arc center and its radius, Computer Vision Graphics and Image Processing, 38, 317-326, 1987

2. S. M. Thomas and Y. T. Chan: A simple approach for the estimation of circular arc center and its radius, Computer Vision Graphics and Image Processing, 45, 362-370, 1989

3. R. Safee-Rad, I. Tchoukanov, B. Benhabib, K. C. Smith: Accurate parameter estimation of quadratic curves from grey level images, CVGIP: Image Understanding, 54(2), 259-274, 1991

4. M. S. Nixon: Improving an extended version of the Hough Transform, Signal Processing, 19, 321-335, 1990

5. C. Kimme, D. Ballard and J. Sklansky: Finding circles by an array of accumulators, Comms of the ACM, 18(2), 120-122, 1975

6. R. S. Conker: A dual plane variation of the Hough Transform for detecting nonconcentric circles of different radii, Computer Vision Graphics and Image Processing, 43, 115-132, 1988

7. J.Porrill: Fitting Ellipses and Predicting Confidence Envelopes using a Bias Corrected Kalman Filter, Image and Vision Computing, 10(5), 37-4155, 1991

8. T. Ellis, A. Abbood and B. Brillaut: Ellipse Detection and Matching with Uncertainty", Image and Vision Computing, 10(5), 271-276, 1992 